\title{
Uso de fotorreatores UV para a remoção de diclofenaco, bezafibrato e etinilestradiol de esgoto tratado em sistema UASB-FBP
}

\author{
Use of UV photoreactors to remove diclofenac, bezafibrate and \\ ethynylestradiol from sewage treated in UASB-TF system
}

\section{Eros de Faria', Diego Roberto de Sousa Lima', Leandro Pablo dos Santos Xavier², Sérgio Francisco de Aquino ${ }^{3}$, Robson José de Cássia Franco Afonso ${ }^{3}$, Carlos Augusto de Lemos Chernicharo ${ }^{4}$, Rafaela Paiva Gomes ${ }^{5}$}

口

\begin{abstract}
RESUMO
Este trabalho avaliou a remoção de diclofenaco (DCF), bezafibrato (BZF) e etinilestradiol (EE2) de efluentes de sistema UASB-FBP (reator anaeróbio de manta de lodo seguido de filtro biológico percolador) em fotorreatores UV de lâmpadas imersas (FRI) e emersas (FRE). Os resultados mostram que baixa eficiência de remoção de tais compostos foi obtida em ambos fotorreatores quando se utilizou baixo tempo de contato ( 10 min) e baixa concentração inicial de fármacos (O,5 $\mu$ g. L.-1 para EE2, e $21 \mu \mathrm{g} \mathrm{L}^{-1}$ para DCF e BZF). O aumento da concentração inicial de DCF (para 20 mg..-1) e do tempo de contato (para 20 min) resultaram em aumento da eficiência de remoção (de 31 para 83\% no FRI e de 36 para 86\% no FRE), indicando que compostos dissolvidos presentes no efluente biológico afetaram adversamente a remoção de fármacos nos fotorreatores devido a competição pela radiação UV incidente. Palavras-chave: fotorreator; fármacos; pós-tratamento; tratamento esgoto sanitário.
\end{abstract}

\begin{abstract}
This paper evaluated the removal of diclophenac (DCF), bezafibrate (BZF) and ethynylestradiol (EE2) from effluent of an anaerobic reactor coupled to a trickling filter (UASB-TF) in two types of UV photoreactors (immerse UV lamps - FRI and emmersed UV lamps - FRE). The results showed low removal efficiencies in both photoreactors when low contact times (10 min) and low initial concentration of such compounds was used (0.5 $\mu \mathrm{g} \cdot \mathrm{L}^{-1}$ for EE2 and $21 \mu \mathrm{g} . \mathrm{L}^{-1}$ for DCF and BZF). An increase in the initial concentration of DCF (to $20 \mathrm{mg}^{\mathrm{L}^{-1}}$ ) and of the contact time (to $20 \mathrm{~min}$ ) led to an increase in the removal efficiency (from 31 to $83 \%$ in FRI and from 36 to $86 \%$ in FRE), suggesting that dissolved compounds present in the biological effluent adversely affected the removal of the pharmaceutical tested due to competition for the incident UV radiation.
\end{abstract}

Keywords: photoreactor. pharmaceuticals; post-treatment; sewage treatment.

\section{INTRODUÇÃO}

O surgimento de novas drogas, cosméticos e produtos de higiene pessoal, associado aos seus maiores usos devido ao crescimento demográfico e melhoria da qualidade de vida da população, tem aumentado a presença de poluentes orgânicos emergentes, tais como fármacos e desreguladores endócrinos, no esgoto sanitário. Embora o interesse científico e conscientização pública pela contaminação ambiental por fármacos remonte à década de 1970, pesquisas de monitoramento de tais contaminantes em águas superficiais, esgotos bruto e tratado só se intensificaram nos países desenvolvidos a partir da década de 1980 (KÜMMERER, 2010), sendo que, no Brasil, pesquisas sobre o tema começaram efetivamente na última década (MELO et al., 2009). As principais rotas de contaminação ambiental por fármacos e desreguladores endócrinos são o lançamento de esgoto tratado e in natura, razão pela qual importa avaliar a eficiência de remoção de tais contaminantes em estações de tratamento de esgoto (ETE). Além disso, é importante desenvolver novas tecnologias para complementar os processos biológicos

\footnotetext{
Trabalho realizado na Universidade Federal de Minas Gerais (UFMG) - Belo Horizonte (MG); e na Universidade Federal de Ouro Preto - (UFOP) - Ouro Preto (MG), Brasil. 'Mestre em Engenharia Ambiental pela UFOP - Ouro Preto (MG), Brasil.

${ }^{2}$ Graduado em Química Industrial pela UFOP - Ouro Preto (MG), Brasil.

${ }^{3}$ Professor Associado da UFOP - Ouro Preto (MG), Brasil.

${ }^{4}$ Professor Titular da UFMG - Belo Horizonte (MG), Brasil.

${ }^{5}$ Mestranda do Programa de Pós-Graduação em Engenharia Ambiental da UFOP - Outro Preto (MG), Brasil.

Endereço para correspondência: Sérgio F. de Aquino - Universidade Federal de Ouro Preto - Campus Morro do Cruzeiro - Departamento de Química, Instituto de Ciências

Exatas e Biológicas - 35400-000 - Ouro Preto (MG), Brasil - Email: sergio@iceb.ufop.br

Recebido: 06/09/12 - Aceito: 29/04/15 - Reg. ABES: 101320
} 
convencionais (por exemplo, reatores anaeróbios de manta de lodo; sistema de lodos ativados; sistema de lagoas) na remoção de fármacos/desreguladores endócrinos.

Alguns estudos reportam efeitos adversos à fauna causados por fármacos/desreguladores endócrinos mesmo quando presentes no ecossistema em concentrações baixas, na faixa de centenas de ng. $\mathrm{L}^{-1}$ a dezenas de $\mu \mathrm{g} . \mathrm{L}^{-1}$, razão pela qual tais compostos são comumente chamados de microcontaminantes orgânicos. Há uma gama de microcontaminantes orgânicos presentes em águas superficiais, esgoto bruto e tratado, sendo os fármacos diclofenaco (anti-inflamatório), bezafibrato (antilipêmico) e etinilestradiol (estrogênio) muito citados na literatura internacional devido ao elevado consumo, elevada persistência ambiental ou elevada toxicidade aquática. Como exemplo, Kümmerer (2009) cita a genotoxicidade e distúrbios endócrinos como alguns dos efeitos observados em peixes expostos ao etinilestradiol (EE2), um estrogênio sintético muito utilizado em pílulas anticoncepcionais. Goodman e Gilman (2006) observaram alterações na transcrição de genes que codificam determinadas proteínas de roedores, supostamente devido ao maior consumo de bezafibrato (BZF) por tais animais. Por sua vez, Coleman et al. (2004) observaram aumento na mortalidade de aves, tais como o abutre oriental de dorso branco, aparentemente associada a doenças renais ocasionadas por resíduos de diclofenaco (DCF) proveniente da alimentação de peixes contaminados.

A presença de BZF, DCF e EE2 no meio ambiente preocupa pelo fato de os mesmos serem pouco voláteis, apresentarem baixa tendência à sorção (exceto etinilestradiol), e serem lentamente (por exemplo, diclofenaco) ou apenas moderadamente biodegradados (JOSS et al., 2005), o que resulta na sua baixa remoção nas ETE convencionais e, consequentemente, na presença de tais microcontaminantes em corpos d'água superficiais. Como exemplo, estudo recente de Brandt (2012) mostrou que o esgoto bruto de Belo Horizonte (MG) contém, em termos medianos, 99,9 ng..-1 de DCF e 94,4 ng.L-1 de BZF, e que sistemas que combinam reatores anaeróbios de manta de lodo (UASB) e unidades de pós-tratamento aeróbio (por exemplo, Filtro Biológico Percolador - FBP), foram pouco eficientes na remoção de diclofenaco e bezafibrato. A remoção de bezafibrato só foi efetiva quando se utilizou um FBP contendo espuma de poliuretano como meio suporte, no sistema denominado Rotosponge ${ }^{\circledast}$ (CHERNICHARO \& ALMEIDA, 2011).

Pelo exposto, este trabalho teve como objetivo principal avaliar a eficiência do processo de fotólise direta (radiação UV) na degradação de diclofenaco, etinilestradiol e bezafibrato presentes em esgoto tratado (efluente de um sistema UASB-FBP), por meio do emprego de fotorreatores de lâmpadas imersas (FRI) e de lâmpadas emersas (FRE).

\section{METODOLOGIA}

Os experimentos foram divididos em três etapas, sendo cada etapa correspondente a uma campanha de coleta, como mostra a Tabela 1. Em todas as etapas, os fotorreatores funcionaram em regime de alimentação contínua, tendo sido operados com água potável ou efluente do sistema UASB-FBP previamente fortificados com os fármacos de interesse (BZF, DCF e EE2).

Em cada condição operacional (etapas de 1 a 3), o reator era alimentado por aproximadamente $20 \mathrm{~min}$ com as lâmpadas acesas antes de se coletar qualquer amostra, com vistas a realizar o aquecimento prévio das lâmpadas e a regulagem da vazão. Após este período, os reatores eram então operados por cerca de $1 \mathrm{~h}$, coletando-se $500 \mathrm{~mL}$ de amostras dos pontos P1 a P3 (ver Figura 1), em intervalos regulares de aproximadamente $10 \mathrm{~min}$.

As amostras coletadas nos pontos P1 a P3 foram inicialmente filtradas em filtros de papel (porosidade de $8 \mu \mathrm{m}$ ) e de fibra de vidro (porosidade de 1,2 $\mu \mathrm{m}$ ) para a remoção de sólidos suspensos, sendo então armazenadas em frascos de vidros âmbar dentro de freezer $\left(-10^{\circ} \mathrm{C}\right)$, até o momento da análise. Foram utilizados frascos de vidro para armazenar as amostras para evitar qualquer tipo de adsorção dos fármacos. Para análise em sistema de cromatografia líquida acoplada a espectrômetro de massas de alta resolução (CLAE-EM-AR, Shimadzu), as amostras aquosas foram submetidas à técnica de extração em fase sólida (exceto na etapa 3) para a concentração dos fármacos de interesse.

O procedimento de extração e análise cromatográfica foi adaptado conforme metodologia validada por Queiroz (2011). Na etapa 3, devido a problemas operacionais no equipamento CLAE-EM-AR, a estimativa de remoção de DCF nos fotorreatores foi feita por meio de leitura da sua absorbância em espectrofotômetro UV-Vis (BEL SP 2000 UV), o que só foi possível a partir da dosagem de elevada concentração (20 mg. $\left.\mathrm{L}^{-1}\right)$ do fármaco. Dessa forma, a etapa 3 permitiu avaliar ainda o desempenho dos fotorreatores quando alimentados com elevada carga de fármacos.

O efluente do UASB-FBP ou água potável (etapa 1) eram armazenados em reservatório de $5 \mathrm{~m}^{3}$ onde preparava-se, sob agitação, o afluente dos fotorreatores, por meio da contaminação prévia da matriz aquosa com os fármacos de interesse. Foram utilizadas pílulas anticoncepcionais (Microvlar ${ }^{\circledast}$ ) como fonte de etinilestradiol, e formas purificadas de bezafibrato (Bezafibrato $200 \mathrm{mg}$, EMS Genérico) e diclofenaco (Clofen S $50 \mathrm{mg}$, Grupo Cimed) para preparo dos afluentes que, por meio de caixa reguladora de vazão, alimentava simultaneamente por gravidade os reatores FRI e FRE (Figura 2). Para preparo do etinilestradiol, as pílulas foram maceradas e dissolvidas em metanol antes de serem adicionadas ao reservatório.

Os fotorreatores FRI e FRE foram confeccionados em alumínio e dimensionados para tratar os esgotos de uma população equivalente a 
250 habitantes. O FRI possuía diâmetro e altura de, respectivamente, 20 e $90 \mathrm{~cm}$, correspondendo a um volume útil de 20,0 L. Tal reator foi equipado com 4 fontes de radiação ultravioleta (UV) (lâmpadas de mercúrio de baixa pressão - modelo G15T8, da marca Phillips), de $30 \mathrm{~W}$ cada. $\mathrm{O}$ espaçamento entre as fontes foi de $6,9 \mathrm{~cm}$ e, para possibilitar a medida a intensidade de radiação que atravessava a fase aquosa, foram instalados 5 visores de quartzo (15 mm de diâmetro e 2,7 mm espessura) em três alturas diferentes, a cada $120^{\circ}$ ao redor do corpo do reator, o que totalizava 15 visores.

Por sua vez, o FRE possuía as seguintes dimensões externas: comprimento de $122 \mathrm{~cm}$, largura de $50 \mathrm{~cm}$ e altura de $14 \mathrm{~cm}$. Internamente, o FRE era subdividido em 4 calhas em paralelo, com as seguintes dimensões: comprimento de $88 \mathrm{~cm}$, largura de $12 \mathrm{~cm}$

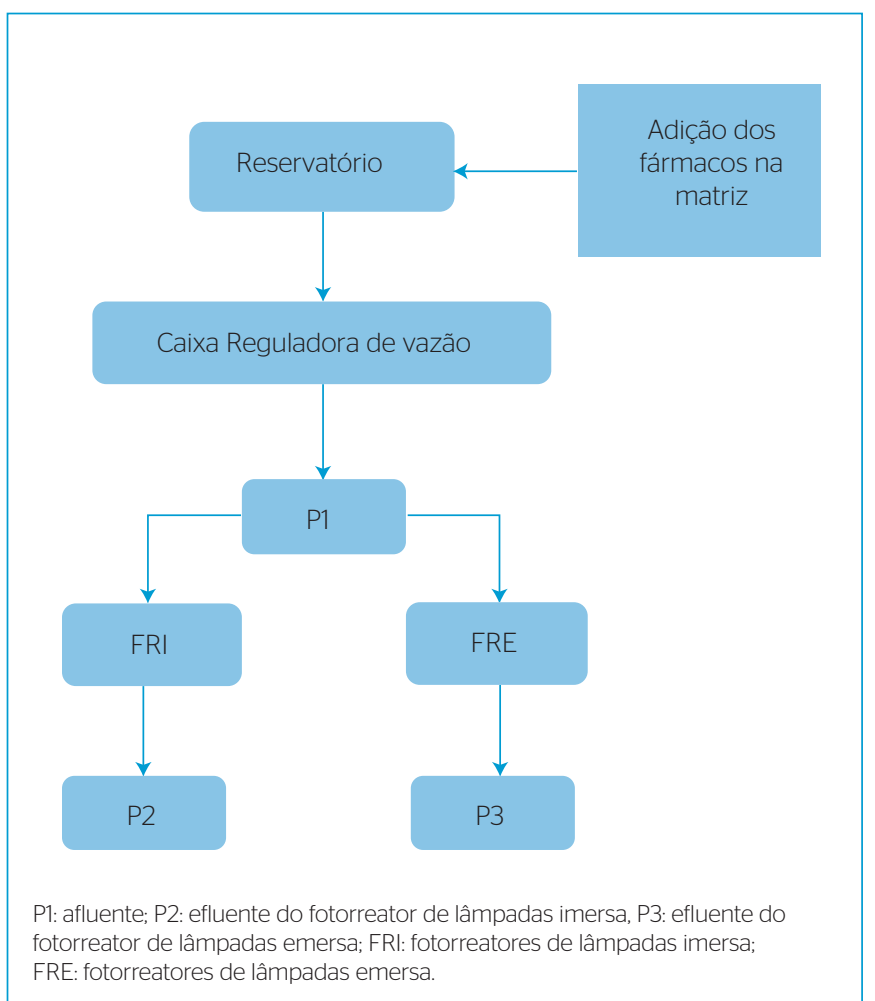

Figura 1 - Fluxograma do tratamento empregado nos fotorreatores com indicação dos pontos de coleta das amostras. e altura da lâmina d'água de $4,7 \mathrm{~cm}$, esta última ajustada por meio de vertedouro. O volume útil do FRE era o mesmo do FRI, equivalente a 20,7 L. Tal reator também foi equipado com quatro fontes de radiação UV (idênticas àquelas utilizadas no FRI). Da mesma forma que no FRI, a medida da intensidade da luz que atravessava o meio líquido se deu por meio de quatro visores de quartzo com $15 \mathrm{~mm}$ de diâmetro e 2,7 mm espessura, instalados na parte mediana inferior de cada uma das quatro calhas do FRE, conforme representado na Figura 3. A leitura da radiação nos visores de quartzo foi feita sempre ao final das coletas, em ambos os fotorreatores, por meio de radiômetro portátil (UV Light Meter) que fora calibrado pelo fabricante.

\section{RESULTADOS E DISCUSSÃO}

$\mathrm{Na}$ Figura 4, são apresentados os resultados referentes à etapa 1, na qual os fotorreatores foram operados com água potável contaminada com os fármacos BZF, DCF e EE2. Os dados obtidos indicam que a eficiência de remoção de DCF foi similar em ambos os fotorreatores, com valores medianos de $47 \%$ no FRE e de $44 \%$ no FRI, sendo que o FRE se mostrou mais robusto que o FRI, o que pode ser percebido pela menor variabilidade dos dados. Tais resultados indicam que a fotólise direta resultou em $\sim 50 \%$ de remoção de DCF em baixo tempo de contato (5 min). Em experimentos com água natural (de lago suíço) fortificada com DCF, Buser (1998) conseguiu degradação quase completa ( 99\%) de DCF $\left(\mathrm{C}_{\mathrm{o}}=100 \mathrm{ng} . \mathrm{L}^{-1}\right)$ com radiação solar, contudo, um tempo de contato de $96 \mathrm{~h}$ (4 dias) foi utilizado. Estrada (2008) também observou redução superior a 70\% na concentração inicial (50 mg. $\mathrm{L}^{-1}$ ) de DCF dissolvido em água destilada, durante 30 horas de exposição à radiação solar, com intensidade estimada em $25 \mathrm{~mW} \cdot \mathrm{cm}^{-2}$, o que totalizava uma dose de radiação de 2.700 W.s.cm ${ }^{-2}$, valor bem superior ao utilizado neste estudo. Segundo esse autor, a absorção de radiação UV pelo DCF provoca foto-alteração da sua estrutura, que acarreta em sua espontânea e rápida transformação. Infelizmente, não foram encontrados artigos que expliquem o mecanismo de transformação ou identifiquem os principais subprodutos formados.

Tabela 1 - Condições impostas aos fotorreatores de lâmpadas imersa e emersa em cada etapa operacional.

\begin{tabular}{|c|c|c|c|c|c|c|c|c|}
\hline \multirow{2}{*}{ Etapa } & \multicolumn{3}{|c|}{ Concentração Inicial Teórica ( $\left.\mu \mathrm{g} . \mathrm{L}^{-1}\right)$ no afluente (AFL) } & \multirow{2}{*}{ Matriz } & \multirow{2}{*}{ TDH (min) } & \multirow{2}{*}{$\begin{array}{c}\text { Turbidez } \\
\text { (NTU) }\end{array}$} & \multicolumn{2}{|c|}{ Dose de Radiação (mW.s.cm²) } \\
\hline & DCF & BZF & EE2 & & & & FRI & FRE \\
\hline 1 & 34,7 & 69,4 & 0,22 & Água* & 5 & $<5$ & - & $192-$ \\
\hline 2 & 21,0 & 21,0 & 0,53 & \multirow{2}{*}{$\begin{array}{l}\text { Efluente de } \\
\text { UASB-FBP }\end{array}$} & 5 & 19 & 30 & 228 \\
\hline 3 & 20.000 & NA & NA & & 10,20 e 40 & 17 & 75 & 271 \\
\hline
\end{tabular}

*utilizou-se água potável que supostamente continha cloro residual cuja concentração não foi determinada; - não medido;

DCF: diclofenaco; BZF: bezafibrato; EE2: etinilestradiol; TDH: tempo de contato; NTU: unidade nefelométrica de turbidez; UASB-FBP: reator anaeróbio de fluxo ascendente seguido de filtro biológico percolador; FRI: fotorreatores de lâmpadas imersa; FRE: fotorreatores de lâmpadas emersa; NA: não se aplica. 

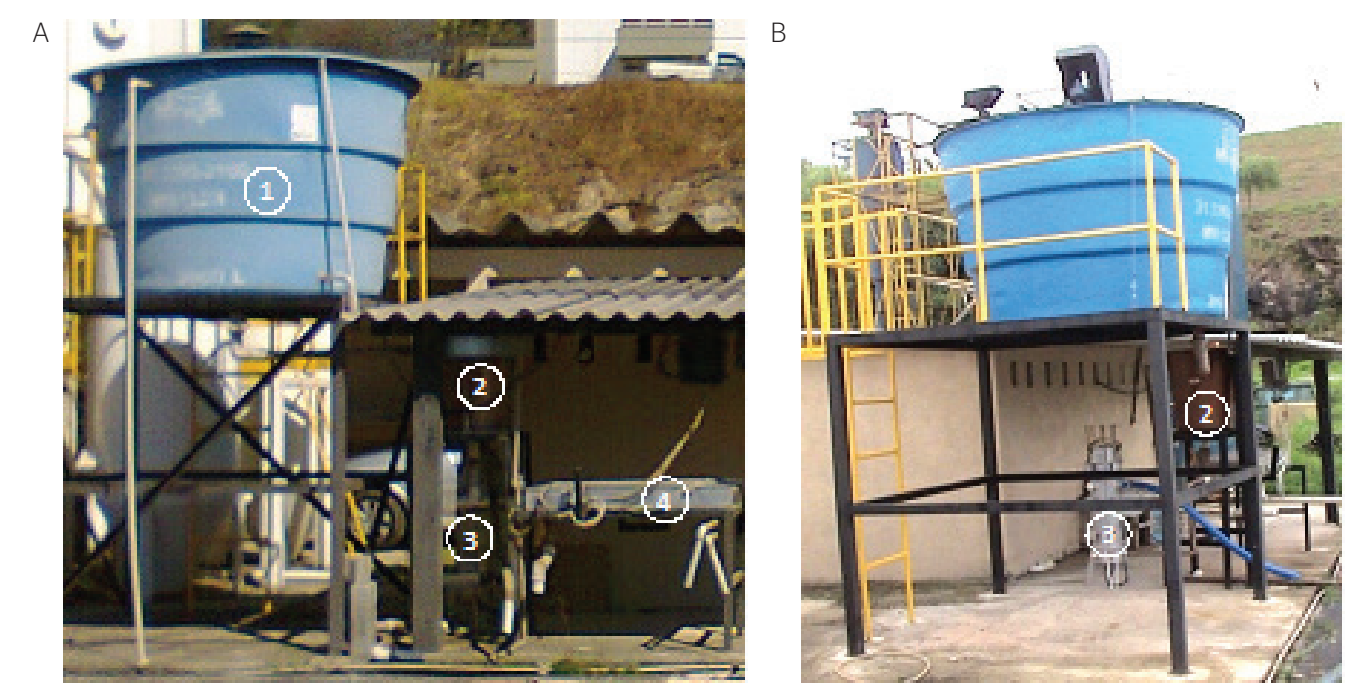

(1) Reservatório

(2) Caixa reguladora de vazão

(3) Fotorreator de lâmpadas imersas

(4) Fotorreator de lâmpadas emersas

Figura 2 - Identificação das unidades que compuseram o aparato experimental utilizado na pesquisa. Vista frontal (A) e lateral (B).

(1) Esgoto Afluente

(2) Fonte de radiação UV

(3) Dispositivo de limpeza

(4) Saída do efluente

(5) Descarga de fundo

(6) Câmara de entrada

(7) Descarte na entrada

(8) Vertedouro de entrada

(9) Calhas

(10) Visores no fundo das calhas

(11) Placa divisora das calhas

(12) Vertedouro de saída
(A)

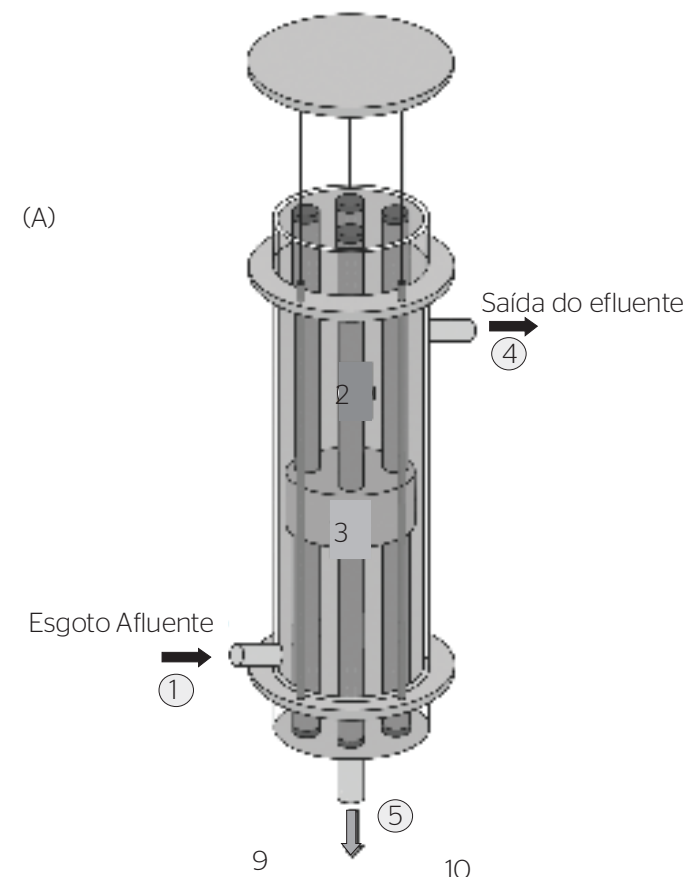

(B)

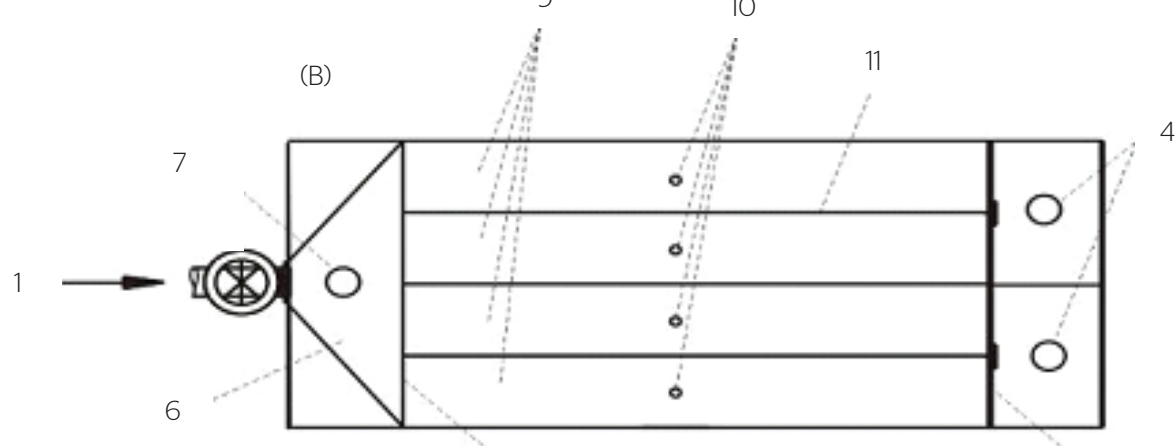

8

12

Figura 3 - Esquema dos fotorreatores fotorreatores de lâmpadas imersa (A) e fotorreatores de lâmpadas emersa (B) construídos e utilizados na pesquisa. 
As concentrações de EE2 na saída dos fotorreatores ficaram abaixo do limite de detecção do equipamento utilizado na análise das amostras. Coleman et al. (2004) mostraram, em estudos de fotólise direta do EE2 sob radiação ultravioleta (UVA, $125 \mathrm{~W}$ ), que a remoção de tal composto foi proporcional à dose de radiação empregada, sendo que eficiências de 50\% na remoção de EE2 foram obtidas com um tempo de contato de 23 min. Quando o tempo de contato foi aumentado para $72 \mathrm{~min}$, a eficiência de remoção subiu para 90\%; contudo, o trabalho de Coleman et al. (2004) não menciona os valores de intensidade da radiação utilizada, para fins de comparação com o presente estudo.

No caso do BZF, os resultados presentes na Figura 4 mostram que uma maior eficiência mediana de remoção foi obtida no FRE (53\%) comparado ao FRI (40\%). Kim et al. (2009), utilizando fotorreator com uma lâmpada imersa (dose de radiação de 1.025 mW.s.cm-2) para remover BZF de efluentes de tratamento secundário, constataram uma eficiência de remoção de $77 \%$, quase o dobro do valor mediano obtido neste estudo para o FRI. Uma possível explicação para tal fato está relacionada ao maior tempo de contato (223 min) utilizado por Kim et al. (2009), que implicou em doses de radiação bem maiores às utilizadas neste estudo.

Em outro estudo, uma solução de bezafibrato foi irradiada com luz solar e as transformações ocorridas na molécula foram monitoradas por cromatografia de camada delgada após coleta de amostras nos tempos de contato de 50, 100 e 200 h. Produtos da fotólise foram devidamente identificados apenas nas amostras coletadas após 200 h de radiação. Neste caso, a fotólise direta levou ao acúmulo de derivados fenólico e éter, respectivamente, pelas perdas de ácido isobutírico e gás carbônico da porção carboxilada da molécula de bezafibrato.

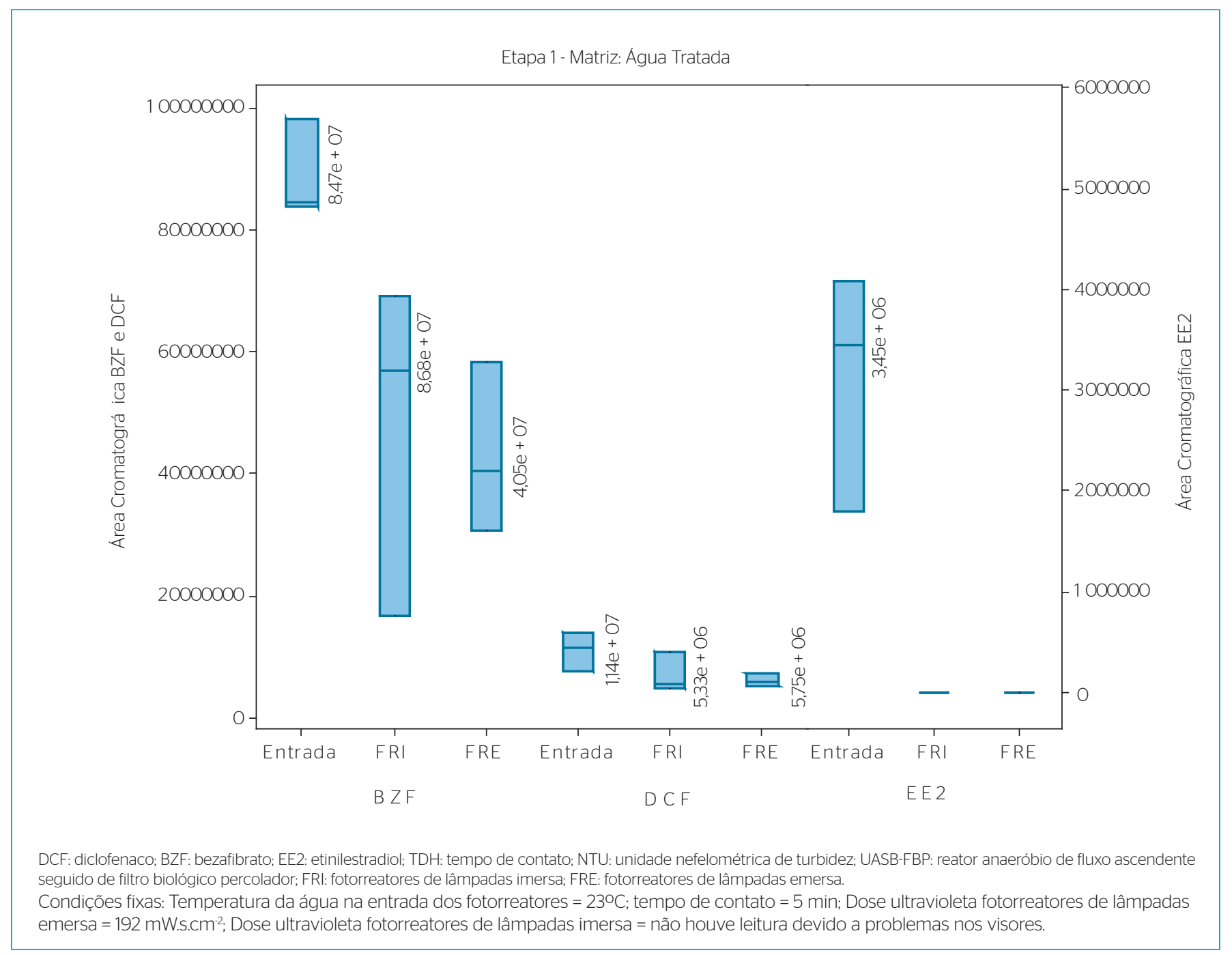

Figura 4 - Remoção de bezafibrato, diclofenaco e etinilestradiol nos fotorreatores na etapa 1. 
De forma geral, era de se esperar um melhor desempenho do FRI em relação ao FRE, uma vez que, no FRI, as lâmpadas ficam em contato direto com o líquido, que tenderiam ainda a aquecê-lo acelerando as reações de fotodegradação. Infelizmente, devido a problemas técnicos nos visores, nesta etapa, não foram realizadas medidas da dose de radiação UV no FRI. De qualquer forma, os resultados mostram que os desempenhos dos FRI e FRE foram semelhantes quando se utilizou água como matriz.

Os resultados observados na etapa 2 são apresentados na Figura 5. Durante esta etapa, utilizou-se efluente do sistema UASB-FBP como matriz aquosa. Ao comparar-se com a etapa anterior, observa-se que a eficiência mediana de remoção do BZF no FRE se manteve próxima a $54 \%$, enquanto que no FRI foi bem maior, cerca de $84 \%$. Apesar de que o esperado fosse redução da eficiência de remoção em ambos os reatores devido à presença de sólidos suspensos, que poderiam causar bloqueio à radiação UV incidente, a maior eficiência obtida no FRI nesta etapa pode ser explicada pelo aumento da radiação incidente, já que foram colocadas lâmpadas novas neste fotorreator no início da etapa 2.

A concentração de EE2 detectada na saída de ambos os reatores foi superior àquela determinada na entrada, sugerindo que houve formação do mesmo durante a fotólise. O aumento da concentração de EE2 observada em ambos os reatores pode ter ocorrido devido ao fenômeno de degradação parcial ou hidrólise de formas conjugadas de estradiol presentes no efluente do UASB-FBP. Formas conjugadas (como sulfato de etinilestradiol, glucuronídeo de etinilestradiol) do hormônio sintético presentes no esgoto bruto não seriam detectadas pelo método de análise desenvolvido (QUEIROZ et al., 2012) e, se não foram eficientemente removidas nos sistemas biológicos de tratamento, elevariam o potencial de formação de EE2 nos fotorreatores. A hipótese aqui é

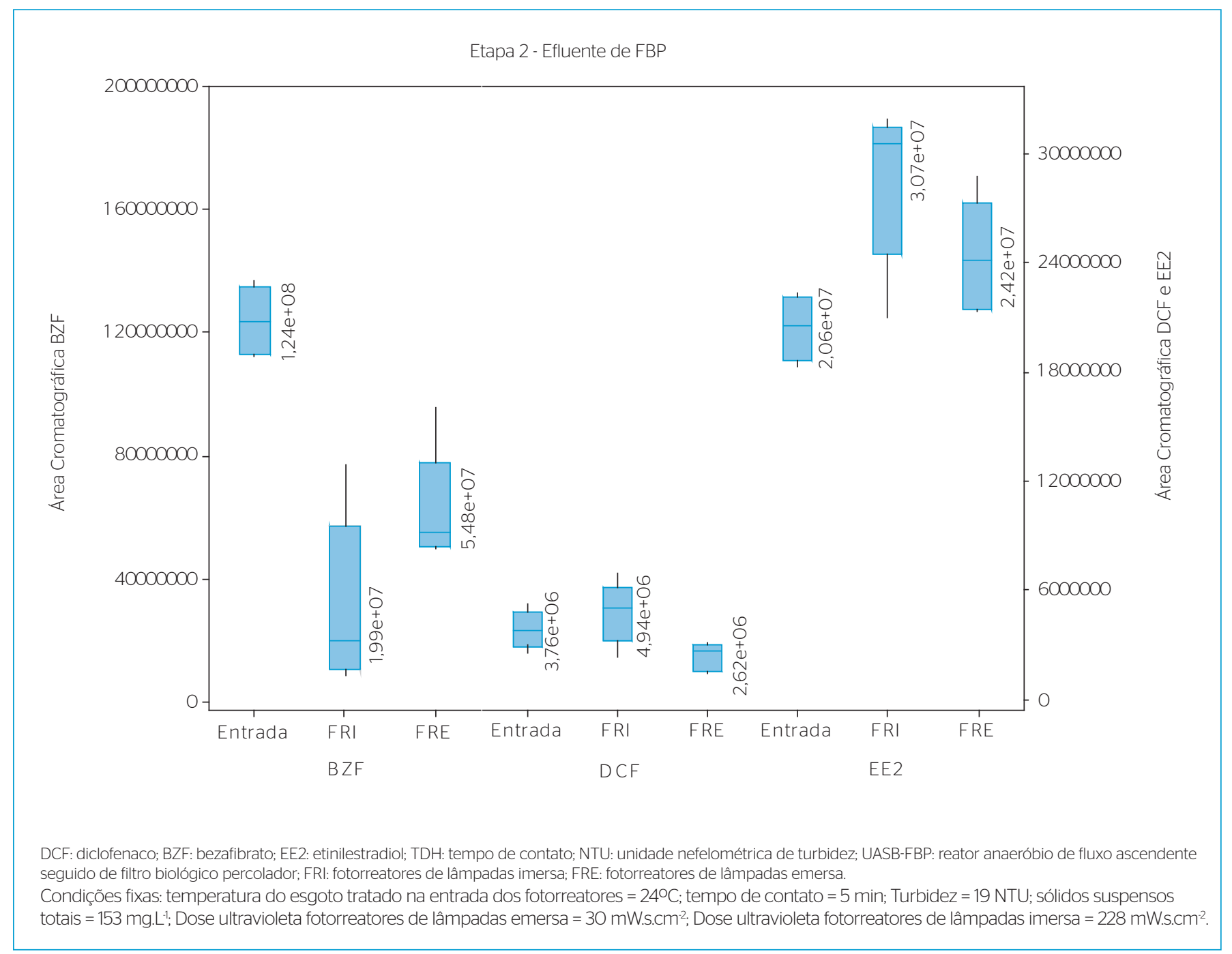

Figura 5 - Remoção de bezafibrato, diclofenaco e etinilestradiol nos fotorreatores na etapa 2. 
que a dose de radiação UV fornecida no fotorreator foi suficiente para converter as formas conjugadas em EE2, mas insuficiente para promover a sua total mineralização devido ao baixo tempo de contato empregado. Dessa forma, uma eventual mineralização do EE2 adicionado seria compensada pela sua produção a partir das formas conjugadas presentes no esgoto bruto.

No caso do DCF a eficiência mediana de remoção no FRE (42,5\%) foi similar ao valor obtido na etapa 1 (47\%), sendo que para o FRI não houve remoção de tal composto na etapa 2. A presença de turbidez (19 NTU) no efluente do UASB-FBP parece ter influenciado a remoção de DCF no FRI uma vez que o fluxo ascensional promovia agitação suficiente para manter os sólidos presentes em suspensão, bloqueando parte da radiação UV incidente. No FRE, isso não foi evidente devido à visível sedimentação dos sólidos suspensos graças à baixa velocidade $\left(\sim 24,0 \mathrm{~cm} \cdot \mathrm{min}^{-1}\right)$ de escoamento nas calhas.

Além disso, pode ter ocorrido liberação de DCF a partir das formas conjugadas, tendo em vista que o DCF é metabolizado e excretado em formas hidroxiladas (BUSER, 1998; GOODMAN \& GILMAN, 2006). Como o método de análise utilizado nesta pesquisa não foi desenvolvido para detectar tais estruturas (QUEIROZ et al., 2012), qualquer mineralização de DCF pode ter sido compensada pela sua produção a partir das formas hidroxiladas, principalmente no FRI onde o bloqueio de parte da radiação incidente pelos sólidos suspensos impossibilitou a completa fotodegradação de tal fármaco.

$\mathrm{Na}$ etapa 3 o fármaco DCF foi adicionado ao efluente do sistema UASB-FBP em concentração mais elevada $\left(20 \mathrm{mg} \cdot \mathrm{L}^{-1}\right)$ para permitir o seu monitoramento por equipamento UV-VIS em razão da impossibilidade de utilização do CLAE-EM-AR devido a problemas técnicos. Dessa forma, a etapa 3 permitiu avaliar a robustez dos fotorreatores e o desempenho de tais sistemas quando alimentados com elevada carga de diclofenaco. Nesta etapa, a concentração de DCF foi monitorada por meio da leitura de sua absorbância na faixa de 273 a $300 \mathrm{~nm}$, previamente identificada como sendo a região de máxima absorção da radiação por tal contaminante.

A Figura 6 apresenta a ocorrência de DCF no afluente e efluente dos fotorreatores, em função do tempo de contato utilizado, em termos deárea do pico de absorção observado. Percebe-se que, para o TDH de $10 \mathrm{~min}$, foram observados 23 e $40 \%$ de remoção de DCF no FRI e FRE, respectivamente,

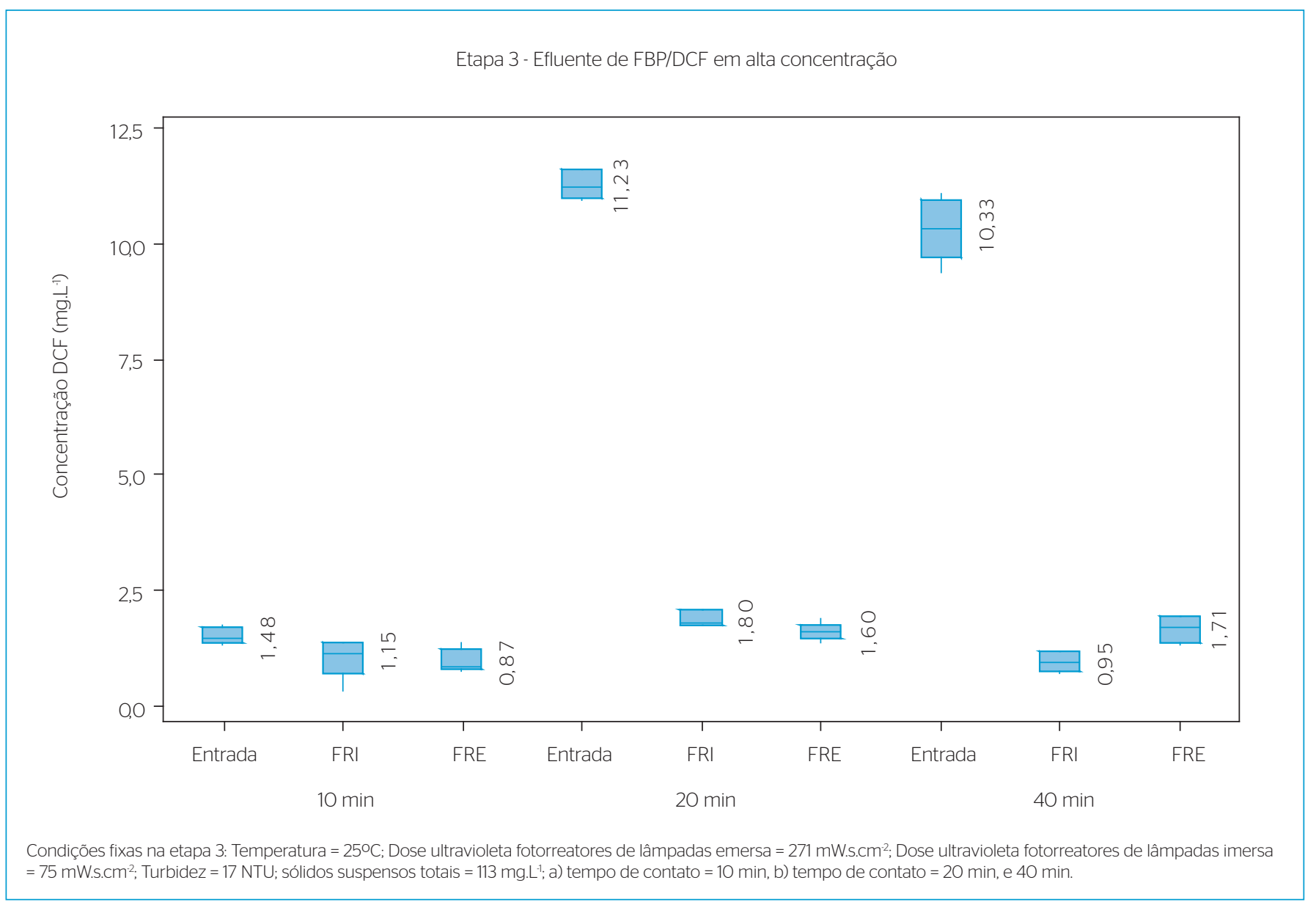

Figura 6 - Área de absorção do diclofenaco obtida por espectroscopia Ultravioleta-Visível. 
sendo que a remoção no FRE foi próxima àquela obtida quando se utilizou tal fármaco em concentração menor (etapa 2). Ao aumentar-se o TDH para $20 \mathrm{~min}$, obtiveram-se eficiências de remoção aproximadamente iguais para o FRI (84\%) e FRE (86\%). Já o aumento do TDH de 20 para $40 \mathrm{~min}$ não gerou melhora significativa na eficiência de degradação de DCF, uma vez que foram observadas eficiências de remoção de 91 e 83\% nos reatores FRI e FRE, respectivamente. Assim, pode-se concluir que o TDH de 20 min é suficiente para garantir resultados satisfatórios em termos de remoção de DCF por fotólise direta. A maior remoção de DCF na etapa 3 quando comparado às etapas 1 e 2 pode ser justificada pela elevada concentração inicial empregada. Como a cinética de fotodegradação de DCF em processo de batelada seguiu ordem 2 (dados não apresentados), esse resultado era esperado, uma vez que a taxa de remoção é proporcional ao quadrado da concentração do fármaco presente.

A Tabela 2 apresenta um resumo dos resultados obtidos em todas as etapas desenvolvidas neste trabalho. O fato de na etapa 1 ter sido utilizada

Tabela 2 - Resumo dos resultados, em termos de porcentagem de remoção, obtidos nas etapas 1, 2 e 3 nos fotorreatores fotorreatores de lâmpadas imersa e fotorreatores de lâmpadas emersa.

\begin{tabular}{|c|c|c|c|c|c|c|c|c|c|c|}
\hline \multirow{3}{*}{ Etapa } & \multirow{3}{*}{ TDH (min) } & \multicolumn{6}{|c|}{ Porcentagem de Remoção (\%) } & \multirow{3}{*}{$\begin{array}{l}\text { Turbidez } \\
\text { (NTU) }\end{array}$} & \multirow{2}{*}{\multicolumn{2}{|c|}{$\begin{array}{l}\text { Dose de Radiação } \\
\left(\mathrm{mW} . \mathrm{s} \cdot \mathrm{cm}^{2}\right)\end{array}$}} \\
\hline & & \multicolumn{2}{|c|}{ DCF } & \multicolumn{2}{|c|}{ BZF } & \multicolumn{2}{|c|}{ EE2 } & & & \\
\hline & & FRI & FRE & FRI & FRE & FRI & FRE & & FRI & FRE \\
\hline 1 & 5 & 35,9 & 45,2 & 46,5 & 51,5 & $\sim 100,0$ & $\sim 100,0$ & $<1$ & - & 192 \\
\hline 2 & 10 & $-23,6$ & 38,9 & 75,1 & 50,0 & $-39,4$ & $-19,2$ & 19 & 30 & 228 \\
\hline \multirow{3}{*}{3} & 10 & 31,0 & 36,0 & NA & NA & NA & NA & \multirow{3}{*}{17} & \multirow{3}{*}{75} & \multirow{3}{*}{271} \\
\hline & 20 & 83,3 & 85,7 & NA & NA & NA & NA & & & \\
\hline & 40 & 90,6 & 83,8 & NA & NA & NA & NA & & & \\
\hline
\end{tabular}

-: não medido; DCF: diclofenaco; BZF: bezafibrato; EE2: etinilestradiol; TDH: tempo de contato; NTU: unidade nefelométrica de turbidez; UASB-FBP: reator anaeróbio de fluxo ascendente seguido de filtro biológico percolador; FRI: fotorreatores de lâmpadas imersa; FRE: fotorreatores de lâmpadas emersa; NA: não se aplica

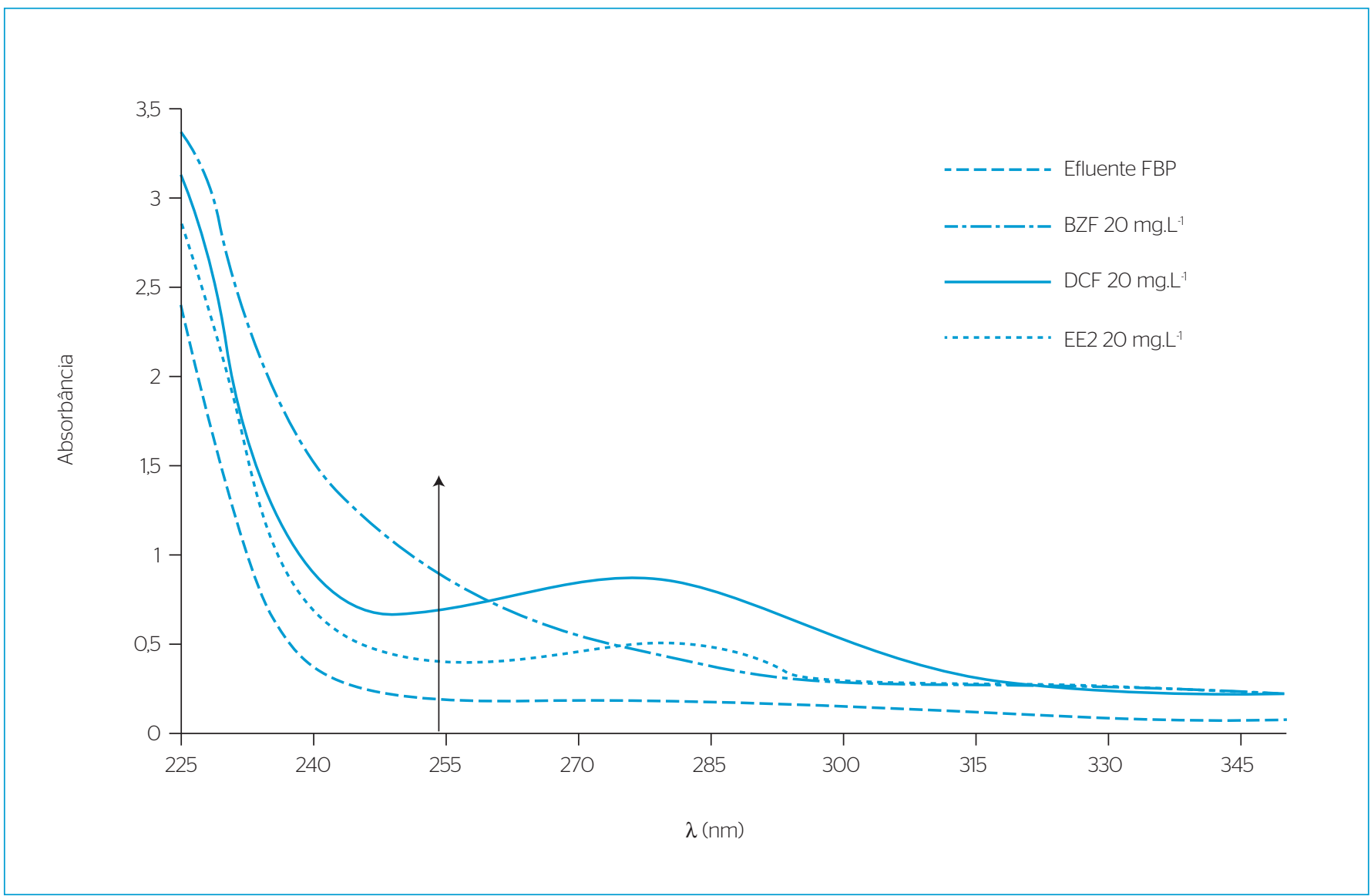

Figura 7 - Espectros de absorbância do efluente do sistema reator anaeróbio de fluxo ascendente seguido de filtro biológico percolador e de soluções de fármacos preparadas nesta matriz aquosa. 
água potável (baixa turbidez) como matriz, mostra que a eventual presença de sólidos pode gerar queda na eficiência de remoção devido ao bloqueio da radiação emitida pela lâmpada. Além disso, o efluente do UASB-FBP apresenta compostos dissolvidos que competem pela radiação UV incidente (Figura 7). A Figura 7 mostra que no comprimento de onda de máxima emissão da lâmpada (254 nm) o efluente do sistema UASB-FBP absorve mais radiação do que os fármacos testados. Isso parece explicar a baixa eficiência de remoção de DCF, BZF e EE2 na etapa 2, quando tais compostos estavam presentes em baixas con-

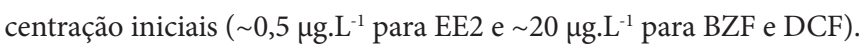
Em outras palavras, a competição da radiação incidente por compostos dissolvidos (como proteínas, surfactantes) que sabidamente absorvem radiação em comprimento de onda próximo àquele de máxima emissão da lâmpada (254 nm), prejudicou a fotólise e degradação dos fármacos que estavam presentes em concentrações da ordem de $\mu$ g. $\mathrm{L}^{-1}$. Desse modo, os dados apresentados na Tabela 2 indicam que os fármacos DCF, BZF e EE2 podem ser eficientemente removidos por fotólise direta se presentes em elevada concentração, uma vez que tal condição permite que eles compitam mais eficientemente pela radiação UV incidente.

\section{CONCLUSÕES}

Os resultados apresentados neste trabalho mostram que fotorreatores de lâmpadas imersas (FRI) e emersas (FRE) foram pouco efetivos na remoção dos fármacos bezafibrato (BZF), diclofenaco (DCF) e etinilestradiol (EE2) presentes em baixas concentrações (da ordem de dezenas $\mu \mathrm{g} . \mathrm{L}^{-1}$ ) em efluente tratado em reator anaeróbio de manta de lodo seguido por filtro biológico percolador (UASB-FBP)

Os resultados mostram ainda que a remoção de DCF, BZF e EE2 fortificados no efluente do UASB-FBP foi menos efetiva quando comparada à água potável fortificada, indicando que compostos dissolvidos e sólidos suspensos presentes no efluente biológico afetaram negativamente a remoção.

Elevada remoção de DCF foi observada em ambos os fotorreatores testados quando a concentração inicial do mesmo era elevada ( 20 mg. $\mathrm{L}^{-1}$ ) indicando que a competição de compostos dissolvidos no efluente do UASB-FBP parece ter limitado a remoção de fármacos quando presentes em baixas concentrações. $\mathrm{O}$ aumento do tempo de contato (TDH) no fotorreator de 10 para 20 min resultou em aumento significativo da eficiência de remoção de DCF, ao passo que o aumento adicional do TDH para 40 min não tenha resultado em ganhos significativos de remoção.

\section{AGRADECIMENTOS}

Os autores gostariam de agradecer ao CNPq, FAPEMIG e CAPES pelo auxílio financeiro para a execução deste trabalho.

\section{REFERÊNCIAS}

BRANDT, E.M.F. (2012) Avaliação da remoção de fármacos e desreguladores endócrinos em sistemas simplificados de tratamento de esgoto (reatores UASB seguidos de pós-tratamento). (Mestrado em Engenharia Ambiental) - Universidade Federal de Minas Gerais, Belo Horizonte (MG).

BUSER, H.R.; POIGER, T.; MÜLLER, M.D. (1998) Occurrence and fate of the pharmaceutical drug diclofenac in surface waters: rapid photodegradation in a lake. Environmental Science \& Technology, v. 32, n. 22, p. 3449-3456

CHERNICHARO, C.A.L. \& ALMEIDA, P.G.S. (2O11) Feasibility of UASB/ trickling filters systems without final clarifiers for the treatment of domestic wastewater in small communities in Brazil. Water Science and Technology, v. 64, p. 1347-1354

COLEMAN, H.M.; ROUTLEDGE, E.J.; SUMPTER, B.R.; EGGINS, B.R.; BYRNE, J.A. (2004) Rapid loss of estrogenicity of steroid estrogens by UVA photolysis and photocatalysis over na immobilised titanium dioxide catalyst. Water Research, v. 38, p. 3233-3240.
ESTRADA, L.A.P. (2008) Degradación Fotoquímica de Contaminantes Emergentes Mediante Processos Solares Evaluácion Analítica. (Doutorado) - Universidad de Almería.

GOODMAN \& GILMAN, A. (2006) As Bases Farmacológicas da Terapêutica. Tradução: Sandra Barreto de Carvalho. The McGraw-Hill Companies. Rio de Janeiro. 11a ed.

JOSS, A.; KELLER, E.; ALDER, A.C.; GOBEL, A.; McARDELL, C.S.; TERNES, T.; SIEGRIST, H. (2005) Removal of pharmaceuticals and fragrances in biological wastewater treatment. Water Research, v. 39, p. 3139-3152.

KIM, I; YAMASHITA, N; TANAKA, H. (2009) Performance of UV and $\mathrm{UV} / \mathrm{H}_{2} \mathrm{O}_{2}$ processes for the removal of pharmaceuticals detected in secondary effluent of a sewage treatment plant in Japan. Journal of Hazardous Materials, n.166, p. 1134-1140.

KÜMERER, K. (2009) The presence of pharmaceuticals in the environment due to human use - present knowledge and future challenges. Journal of Environmental Management, v. 90, p. 2354-2366. 
KÜMERER, K. (2010) Pharmaceuticals in the Environment. Annual Review of Environment and Resources, v. 35, p. 57-76.

MELO, S.A.S.; TROVO, A.G.; BAUTITZ, I.R.; NOGUEIRA, R.F.P. (2009) Degradação de fármacos residuais por processos oxidativos avançados. Química Nova, v.32, n.1, p. 188-197.

QUEIROZ, F.B. (2O11) Desenvolvimento e validação de metodologia para determinação de fármacos e perturbadores endócrinos em amostras de esgoto utilizando extração em fase sólida e cromatografia líquida acoplada à espectrometria de massas. (Mestrado em Engenharia Ambiental) - Universidade Federal de Ouro Preto, Ouro Pretp (MG)

QUEIROZ, F.B.; BRANDT, E.M.F.; AQUINO, S.F.; AFONSO, R.J.C.F.; CHERNICHARO, C.A.L. (2012) Occurrence of pharmaceuticals and endocrine disruptors in raw sewage and their behavior in UASB reactors operated at different hydraulic retention times. Water Science and Technology. v.66, n. 12, p. 2562-2565. 


\section{Errata}

No artigo Uso de fotorreatores UV para a remoção de diclofenaco, bezafibrato e etinilestradiol de esgoto tratado em sistema UASB-FBP, com número de DOI: 10.1590/S1413-41522015020000101320, publicado no periódico Revista Engenharia Sanitária e Ambiental, 20(3):493-502, na página 493 faltou mencionar um autor, junto com sua titulação:

Rafaela Paiva Gomes ${ }^{5}$

${ }^{5}$ Mestranda do Programa de Pós-Graduação em Engenharia Ambiental da UFOP - Outro Preto (MG), Brasil. 